\title{
Wirkfaktoren im Coaching
}

\section{Astrid Schreyögg}

Wie wirkt eigentlich Coaching? Im Zuge der Professionsentwicklung stellt sich diese Frage immer dringlicher. Wir hatten großes Glück beim „Eintreiben“ von Aufsätzen, denn zum Thema ,Wirkfaktoren im Coaching“ konnten wir eine ganze Reihe von Autoren finden, die sich zum Teil schon seit längerer Zeit mit solchen Fragen befassen. Und diese führen sogar einen kritischen Diskurs untereinander.

Im ersten Betrag geben Siegfried Greif, Frank Schmidt und Andre Thamm einen Überblick über den Stand der Forschung zu Wirkfaktoren im Coaching. Forschungstechnisch beruft man sich dabei jeweils auf die Wirkfaktoren, die einstens Klaus Grawe und seine Mitarbeiter für die Psychotherapie-Forschung bemüht hatten. Das sind im Prinzip die Basisvariablen aus der Schule von Rogers und Variablen aus der kognitiven Verhaltenstherapie. So wurden dann auch fürs Coaching Wertschätzung und emotionale Unterstützung seitens des Coachs sowie Ressourcenaktivierung als Hilfe zur Selbsthilfe und Begleitung bei der Umsetzung als relevante Wirkfaktoren benannt.

Peter Behrendt präsentiert nachfolgend sein „Freiburger Erfolgsfaktoren-Coaching“. Der Autor knüpft ebenfalls an die Forschung von Grawe an und leitet daraus vier erfolgskritische Faktoren ab: Ressourcenaktivierung incl. der Coaching-Beziehung, Klärung, Bewältigung und Problemaktualisierung.

Im dritten Beitrag beschäftigt sich Theresa Wechsler mit den Wirkungen, die den sogenannten Coaching-Tools innewohnen. Diese hat Christopher Rauen in mittlerweile drei Bänden herausgegeben. Im Anschluss an die Forschung von Grawe und Greif entwickelt die Autorin ein ,coachingtool-spezifisches“ Wirkfaktorenmodell, mit dem sie dann die Wirkungen einzelner Coaching-Tools aus den beiden Tool-Büchern von Rauen systematisiert. Damit überschreitet sie sogar die bisherige Debatte um die Wirkfaktoren von Coaching. Die Autorin stellt auch die Frage, ob Wirkungsfaktoren fürs Coaching ausschließlich der Psychotherapieforschung entlehnt werden müssen. Wahrscheinlich sollte man mit übergeordneten Konzepten operieren.

\footnotetext{
Online publiziert: 31.10 .2012

(C) Springer Fachmedien Wiesbaden 2012

Dr. A. Schreyögg $(\bowtie)$

Breisgauer Str. 29, 14129 Berlin, Deutschland

E-Mail: info@schreyoegg.de
} 
Jens Kleinert und Sara Wippich beschäftigen sich mit „Vertrauen als Merkmal von Beziehungsqualität“، Diese Autoren betrachten Coaching im Sport, genauer sie reflektieren die Betreuung von Athleten. Sie entwickelten ein Modell, in dem interpersonales Vertrauen als Konsequenz der Evaluation personaler und aufgabenbezogener Betreuungsbedingungen gesehen wird. Wohlwollen und Wertschätzung waren zentrale Bedingungen fürs Vertrauen, während Expertise des Betreuers eine geringere Rolle zu spielen schien.

Zwei Praxisberichte entstammen pädagogischen Feldern. Der erste von Christine Gasser beschäftigt sich mit der ,Evaluation von Supervisionsprozessen an Südtiroler Schulen“. Die Autorin ist „Verantwortliche für den Bereich Supervision und Coaching im Unterstützungssystem des Deutschen Schulamtes“ in Bozen. Auf der Basis von fast 500 befragten Supervisanden kann sie zeigen, dass die Schulen der deutschen Sprachgemeinschaft in Südtirol von der Supervision enorm profitiert haben. Susanne Vaudt und Annelie Nolte untersuchten demgegenüber Wirkprozesse kollegialer Beratung bei KitaLeitungskräften. Die narrativen Interviews zeigten positive Wirkungen im Hinblick auf die Leitungskompetenz. Schließlich präsentieren Matthias Schmidt und Lothar Bildat ein Projekt zur beruflichen Integration von langzeitarbeitslosen jungen Menschen in Sachsen, das auch individuelles Coaching umfasst. Eine umfangreiche Evaluation zeigt nicht nur gute Erfolge im Hinblick auf die berufliche Eingliederung, sondern auch auf die seelische Gesundheit der Teilnehmer/innen. Wir haben diese Arbeit als weiteren Beitrag zur Wirksamkeitsforschung in dieses Heft aufgenommen, obwohl das darin verwendete CoachingKonzept von dem Coaching-Begriff abweicht, wie er in dieser Zeitschrift in der Regel vertreten wird, nämlich als Beratung, Begleitung und Unterstützung von Personen mit Führungs- und Steuerungsfunktionen.

Der Diskurs von Mirco Zwack, Audris Muraitisund Jochen Schweitzer-Rothers ist eine Replik auf die Replik von Ferdinand Buer im Heft 2/12 dieser Zeitschrift zu einem Beitrag der drei AutorInnen in Heft 4/2011. Hier geht es letztlich um normative Fragestellungen zwischen Autoren, die der Humanistischen Psychologie nahestehen, und solchen, die sich aus der Familientherapie kommend mit konstruktivistischen und paradoxen Argumenten „munitionieren“. Wie so oft, stockt hier die Verständigung. Wir freuen uns aber, dass die Autoren ihren ,Schlagabtausch“ in der OSCführen, weil dadurch die Argumentationsmuster für viele andere zugänglich werden. 